\title{
Pain and swelling in the hands and wrists of a 45-year-old woman
}

\author{
Michelle Jung MD, Lillian Barra MD MPH
}

$\Lambda$ previously healthy 45 -year-old woman presents to her doctor's office with fatigue and pain in her fingers and wrists associated with one hour of morning stiffness occurring over several months. The pain is partially relieved by over-the-counter nonsteroidal anti-inflammatory medications. She has had no recent infections or systemic symptoms. On examination, she has bilateral swelling and tenderness in all metacarpophalangeal joints and both wrists.

\section{What is the most likely diagnosis?}

The most likely diagnosis is rheumatoid arthritis. A thorough history and physical examination are important in distinguishing rheumatoid arthritis from other causes of polyarthritis, such as systemic lupus erythematosus and other connective tissue diseases, spondyloarthritis with peripheral joint involvement, joint infections, crystalline arthritis and osteoarthritis. Rheumatoid arthritis should be suspected on the basis of clinical findings: bilateral and symmetric peripheral joint pain and swelling, particularly in small joints, and morning stiffness for more than 30 minutes. ${ }^{1}$ The classification criteria for rheumatoid arthritis were revised in 2010 to increase the chances of early detection (Box 1). ${ }^{1}$ In a study involving 2258 patients with early rheumatoid arthritis, the new criteria had a sensitivity of $71 \%-84 \%$ and a specificity of $60 \%-$ $74 \%$ for detection. ${ }^{2}$ This implies that there are risks of false-negative and false-positive diagnoses, particularly when the probablity of having the disease is low in a primary care setting. Therefore, the criteria may serve as a guide but should not be used in isolation.

Canadian consensus statements, supported by findings from cohort studies and randomized controlled trials, stress the importance of early diagnosis of rheumatoid arthritis and prompt initiation of disease-modifying antirheumatic drugs to prevent long-term damage and disability. ${ }^{3,4}$
What laboratory tests should be ordered? Consensus statements recommend measuring the erythrocyte sedimentation rate or the C-reactive protein level or both in cases of suspected rheumatoid arthritis; however, these tests are nonspecific. ${ }^{1,4}$ Detecting the presence of the antibodies associated with rheumatoid arthritis, such as rheumatoid factor (RF) and anticitrullinated peptide antibody (commonly measured as anticyclic citrullinated peptide antibody [anti-CCP2]) is also recommended to aid in diagnosis. ${ }^{1}$

Systematic reviews of the diagnostic value of antibodies in undifferentiated inflammatory arthritis showed that antibodies associated with rheumatoid arthritis are predictive of the disease (positive likelihood ratio 1.1-13.5 for rheumatoid factor and 12.7 for anti-CCP2). ${ }^{5,6}$ However, rheumatoid factor can be found in other conditions, such as bacterial endocarditis, hepatitis $\mathrm{C}$ virus infection and primary biliary cirrhosis, whereas anti-CCP2 has a higher specificity for rheumatoid arthritis (96\% v. 86\%). ${ }^{6}$ Testing for anti-CCP2 and rheumatoid factor is not helpful for ruling out disease, because up to $50 \%$ of patients with rheumatoid arthritis are antibody-negative. ${ }^{6}$ It should be performed only if persistent synovitis is present, and antibody status should not be used in isolation to rule in or rule out the disease. ${ }^{7}$

\section{Is radiography necessary?}

Radiography in patients with early rheumatoid arthritis may only show nonspecific findings, such as osteopenia. However, the presence of erosions typical of rheumatoid arthritis is diagnostic. ${ }^{3}$ Thus, radiographs of the hands and feet should be done when inflammatory arthritis is suspected, as recommended by the Canadian Rheumatology Association consensus statement. ${ }^{4}$

\section{Should this patient be referred to an arthritis care specialist?}

Even if a patient does not meet the classification criteria for rheumatoid arthritis, referral to an arthri-
Competing interests: None declared.

This article has been peer reviewed.

Correspondence to: Michelle Jung, michelle .jung@londonhospitals.ca

CMAJ 2015. DOI:10.1503 /cmaj.131017 
tis care specialist may be warranted. A Canadian consensus statement stresses that identification of persistent synovitis (duration $\geq 6 \mathrm{wk}$ ) on examination remains the most reliable diagnostic finding for patients requiring urgent referral, even if they do not meet the diagnostic criteria. ${ }^{3}$ A referral should also be made if other types of inflammatory arthritis are suspected based on clinical assessment (i.e., inflammatory back pain or multiorgan disease).

\section{Case revisited}

The patient's laboratory test results showed elevated C-reactive protein, rheumatoid factor and

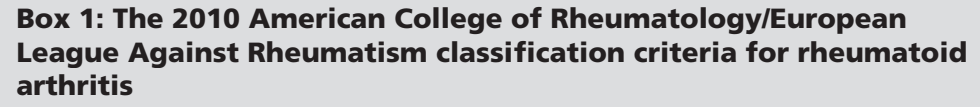

Target population: patients who

1) have at least 1 joint with definite clinical synovitis (swelling),

2) with the synovitis not better explained by another disease

Classification criteria (score-based algorithm: add score of categories A-D; a score of $\geq 6 / 10$ is needed for classification of a patient as having definite rheumatoid arthritis)*

Score

(A) Joint involvement†

1 large joint 0

2-10 large joints

1

1-3 small joints (with or without involvement of large joints)

4-10 small joints (with or without involvement of large joints)

$>10$ joints (at least 1 small joint)

2

3

(B) Serology ( $\geq 1$ test result is needed for classification)‡

Negative RF and negative ACPA

Low-positive RF or low-positive ACPA

0

High-positive RF or high-positive ACPA

2

(C) Acute-phase reactants ( $\geq 1$ test result is needed for classification) Normal CRP and normal ESR Abnormal CRP or abnormal ESR

(D) Duration of symptoms

$<6$ wk 0

$\geq 6 \mathrm{wk}$ 1

Note: $\mathrm{ACPA}=$ anticitrullinated peptide antibody, CRP = C-reactive protein, ESR = erythrocyte sedimentation rate, $\mathrm{RF}=$ rheumatoid factor.

*Although patients with a score of $<6 / 10$ are not classifiable as having rheumatoid arthritis, their status can be reassessed and the criteria might be fulfilled cumulatively over time.

†"Large joints" refers to shoulders, elbows, hips, knees and ankles.

\#"Negative" refers to values that are less than or equal to the upper limit of normal (ULN) for the laboratory and assay; "low-positive" refers to values that are higher than the ULN but less than or equal to three times the ULN; "high-positive" refers to values that are greater than three times the ULN.

Reprinted, with permission, from Aletaha et al. ${ }^{1}$
anti-CCP2 levels that were more than three times the upper limit of normal. No erosive change was noted on radiographs. The total score using the 2010 classification criteria was 8 (3 points for involvement of 4-10 small joints; 3 for high-positive rheumatoid factor or anti-CCP2 value; 1 for abnormal C-reactive protein level or erythrocyte sedimentation rate; and 1 for duration $\geq 6 \mathrm{wk}$ ). The patient was referred to an arthritis care specialist, and therapy with disease-modifying antirheumatic drugs was started.

\section{References}

1. Aletaha D, Neogi T, Silman AJ, et al. 2010 Rheumatoid arthritis classification criteria: an American College of Rheumatology /European League Against Rheumatism collaborative initiative. Ann Rheum Dis 2010;69:1580-8.

2. van der Linden MP, Knevel R, Huizinga TWJ, et al. Classification of rheumatoid arthritis: comparison of the 1987 American College of Rheumatology criteria and the 2010 American College of Rheumatology/European League Against Rheumatism criteria. Arthritis Rheum 2011;63:37-42.

3. Bykerk VP, Baron M, Boire G, et al. Canadian consensus statement on early optimal therapy in early rheumatoid arthritis. New Market (ON): Canadian Rheumatology Association; 2004. Available: http://rheum.ca/images/documents/Canadian_ Consensus_Statement_on_Early_Optimal_Therapy_in_Early_RA .pdf (accessed 2014 July 21)

4. Bykerk VP, Ahhavan P, Hazlewood GS, et al. Canadian Rheumatology Association recommendations for pharmacological management of rheumatoid arthritis with traditional and biologic disease-modifying antirheumatic drugs. J Rheumatol 2012; 39:1559-82.

5. Schoels M, Bombardier C, Aletaha D. Diagnostic and prognostic value of antibodies and soluble biomarkers in undifferentiated peripheral inflammatory arthritis: a systematic review. J Rheumatol Suppl 2011;87:20-5.

6. Whiting PF, Smidt N, Sterne JAC, et al. Systematic review: accuracy of anti-citrullinated peptide antibodies for diagnosing rheumatoid arthritis. Ann Intern Med 2010;152:456-64.

7. Miller A, Mahtani KR, Waterfield MA, et al. Is rheumatoid factor useful in primary care? A retrospective cross-sectional study. Clin Rheumatol 2013;32:1089-93.

Affiliations: Department of Rheumatology (Jung, Barra), Schulich School of Medicine and Dentistry, London, Ont.

Contributors: Both authors contributed substantially and equally to the conception of the article, review of the literature and manuscript revision. They approved the final version submitted for publication and agreed to act as guarantors of the work.

\section{Patient and physician resources}

The Canadian Arthritis Society offers information on different types of arthritis and a network of support groups for patients (available at www.arthritis.ca) 